\title{
Ärztestatistik: Schaffen die Hausärzte die Arbeit noch?
}

_ Die Zahl der Hausärzte hat sich in den Jahren 2013-2014 um die Zahl 54.000 eingependelt, wenn man die Kinderärzte einmal außer Acht lässt. Dabei nahm die Zahl der hausärztlichen Vertragsärzte um etwa $1.400 \mathrm{ab}$, während angestellte Hausärzte im gleichen Umfang hinzukamen.

Ein ähnlicher Umbau findet bei den verschiedenen Arztgruppen im hausärztlichen Bereich statt: Ein Zuwachs an Hausarzt-Internisten kompensiert den Rückgang bei Allgemeinärzten und Praktikern. So geht auch der Rückgang bei den Hausärzten mit eigenem Kassensitz im Wesentlichen auf die Allgemeinmediziner und an zweiter Stelle auf die Praktiker zurück.

In allen drei Gruppen wird die Anstellung beliebter. Diese große Verschiebung führte $2015 \mathrm{zu}$ einer signifikanten Abnahme des hausärztlichen Arbeitsvolumens insgesamt. Wenn man die von der KBV angegebenen Stundenkorridore für hausärztliche Vertragsärzte und angestellte Hausärzte zugrunde legt, ergibt sich ein Rückgang des hausärztlichen Arbeitsvolumens von etwa 37.500 Stunden pro Woche. Um diesen Rückgang zu kompensieren, würde man 670 Vollzeitärzte bei 56 Stunden Vollzeittätigkeit benötigen.

$\mathrm{Da}$ aber meist in Teilzeit arbeitende, angestellte Hausärzte neu ins KV-System kommen, wird prospektiv eine weit höhere Personenzahl benötigt, um das zurückgegangene Arbeitsvolumen zu kompensieren. Weitere Kompensationsfaktoren wie eine geringere Zahl an Arzt-Patienten-Kontakten und oder die Delegation von Aufgaben an qualifizierte Hilfskräfte wie VERAH und NäPa sowie der Einsatz von IT-Systemen sind nicht erfasst.

Interessant ist auch ein Blick auf die Entwicklung bei der Weiterbildung zum Fach Allgemeinmedizin. Offensichtlich kommen aktuell mehr Allgemeinmediziner ins KV-System, als im jeweiligen Jahr diese Fachrichtung abgeschlossen hat. Die KBV schätzt, dass nur 70-80\% der Absolventen sich tatsächlich ins KVSystem begeben. Vermutlich gibt es aber einen mehr oder weniger großen Pool von Hausärzten, die zunächst außerhalb des KV-Systems arbeiten.

\section{MMW-KOMMENTAR}

Aller Voraussicht nach wird die Zahl der angestellten Hausärzte absolut und relativ zunehmen. Unter sonst gleichen Bedingungen führt die Zunahme von angestellten Ärzten zu einem Rückgang des Arbeitszeitvolumens. Mittelfristig wird eine große Zahl von älteren Hausärzten in Ruhestand gehen. Deren gleichzeitiger Ersatz durch eine ausreichende Zahl von Zugängen ist fraglich. Die Sicherstellung des notwendigen hausärztlichen Arbeitsvolumens im Verhältnis zum steigenden Bedarf wird schwieriger. Die Gefahr, dass sogenannte "grundversorgende" Facharztgruppen Zugang zur hausärztlichen Tätigkeit erlangen, ist real. Ebenso real ist die Aussicht, dass sich das auf das Honorargefüge auswirken wird.

Immer mehr angehende Hausärzte streben eine Anstellung an. 